\title{
The 35-kilodalton protein gene (p35) of Autographa californica nuclear polyhedrosis virus and the neomycin resistance gene provide dominant selection of recombinant baculoviruses
}

Robert A.Lerch and Paul D.Friesen*

Institute for Molecular Virology and Department of Biochemistry, The Graduate School and College of Agricultural and Life Sciences, University of Wisconsin-Madison, Madison, WI 53706-1596, USA

Received February 3, 1993; Revised and Accepted March 11, 1993

\begin{abstract}
Autographa californica nuclear polyhedrosis virus (AcMNPV) recombinants were constructed to test the effectiveness of the ACMNPV 35-kilodalton protein gene (35K gene) and the bacterial neomycin resistance gene (neo) as dominant selectable markers for baculoviruses. Insertion of the AcMNPV apoptosis suppressor gene (p35) into the genome of p35-deletion mutants inhibited premature host cell death and increased virus yields up to 1200-fold at low multiplicities in Spodoptera frugiperda (SF21) cell cultures. When placed under control of an early virus promoter, the bacterial neomycin resistance gene (neo) restored multiplication of AcMNPV in the same cells treated with concentrations of the antibiotic G418 that inhibited wild-type virus growth greater than 1000 -fold. The selectivity of these dominant markers was compared by serial passage of recombinant virus mixtures. After four passages, the proportion of p35-containing virus increased as much as 2,000,000-fold relative to deletion mutants, whereas the proportion of neo-containing viruses increased 500 -fold relative to wild-type virus under G418 selection. The strength and utility of p35 as a selectable marker was further demonstrated by the construction of AcMNPV expression vectors using polyhedrin-based transfer plasmids that contain p35. Recombinant viruses with foreign gene insertions at the polyhedrin locus accounted for 15 to $30 \%$ of the transfection progeny. The proportion of desired viruses was increased to greater than $90 \%$ by linearizing the parental virus DNA at the intended site of recombination prior to transfection. These results indicate that p35 and neo facilitate the selection of baculovirus recombinants and that p35, in particular, is an effective marker for the generation of ACMNPV expression vectors.
\end{abstract}

\section{INTRODUCTION}

Autographa californica nuclear polyhedrosis virus (AcMNPV) and other members of the family Baculoviridae have attracted considerable attention due to their roles as eukaryotic expression vectors and engineered biological insecticides (for recent reviews, see references 1-5). AcMNPV possesses a complex genome consisting of a circular, double-stranded DNA $(\sim 131-\mathrm{kb})$ that encodes 70 to 100 genes. Only a limited number of these genes have been characterized with respect to their role in virus multiplication, high level protein production, and pathogenesis in insects. An important approach for molecular studies of AcMNPV gene function has been the generation of recombinant viruses that contain mutations or gene replacements. New or altered genes are introduced into the virus genome by allelic replacement, a process by which viral DNA sequences are replaced with transfected plasmid sequences through homologous recombination. The same approach is used to construct recombinant virus vectors that carry a foreign gene inserted under control of a strong virus promoter for high level protein expression $(3,4)$. Since mutant or recombinant viruses are recovered amid a high background of parental virus, a limitation to allelic replacement has been the lack of dominant genes that provide a selectable growth advantage to the desired virus. Such genes would facilitate the amplification and identification of AcMNPV recombinants and are also essential for the isolation of rare virus mutants. Towards the goal of identifying such dominant selectable markers, we have examined two functionally unrelated genes, the AcMNPV 35-kDa protein gene ( $p 35)$ and the bacterial neomycin resistance gene (neo), for their effectiveness in the selection and isolation of recombinant baculoviruses.

p35, encoded by the EcoRI-S genome fragment of AcMNPV, is required for wild-type levels of virus multiplication in a cell line-specific manner (6-8). Although the exact function of $p 35$ is unknown, it directly or indirectly suppresses premature cell death that is the result of apoptosis (6), a host-mediated process

\footnotetext{
* To whom correspondence should be addressed
} 
that involves cell lysis and destruction of host DNA. Cultured $S$. frugiperda (SF21) cells are susceptible to virus-induced apoptosis, whereas Trichoplusia ni (TN368) cells are not. Production of the budded form of the virus (BV) by $p 35$-deletion mutants in SF21 cells is greatly reduced and formation of occluded virus particles is blocked. Subsequent insertion of $p 35$ at an alternate site within the genome of deletion mutants restores BV production and occlusion-specific gene expression to wildtype levels (8). This finding suggested that $p 35$ could be used as a dominant selectable marker for recombinant baculoviruses.

In contrast to virus-encoded $p 35$, neo represents a classical drug resistance marker for dominant selection. This bacterial gene encodes a phosphotransferase that inactivates the protein synthesis inhibitor G418, an aminoglycosidic antibiotic that interferes with eukaryotic 80 S ribosome function $(9,10)$. Insertion of neo into the genome of several vertebrate DNA viruses (including vaccinia virus and Epstein-Barr virus) confers dominant selectability in the presence of drug $(11-13)$. In addition, neo has been used to develop G418-resistant cells lines derived from invertebrates $(14,15)$, including the moth $S$. frugiperda which is a permissive AcMNPV host. This suggested that AcMNPV-mediated expression of neo would also provide drug resistance and thereby facilitate virus selectivity.

We report here that both $p 35$ and neo (in conjunction with G418) provide a selectable advantage to AcMNPV recombinants. Representing the stronger of the two genetic markers, $p 35$ readily permitted the selective amplification of recombinant viruses in mixtures that contained a $10^{6}$-fold excess of virus lacking the gene. This demonstrated that it should be possible to select and subsequently identify rare virus mutants containing an inserted copy of this marker. The strength of selectability of $p 35$ was further utilized for the construction of recombinant viruses by incorporating the marker into allelic transfer plasmids containing a foreign passenger gene. A high proportion of the virus progeny generated after cotransfection with a $p 35$-deletion virus consisted of recombinant viruses with the foreign gene inserted at the proper genomic site. Thus, as dominant selectable markers, both neo and $p 35$ have direct application for the isolation of recombinant baculoviruses. As the strongest dominant marker yet identified for AcMNPV, $p 35$ should be especially useful in facilitating the construction and isolation of virus expression vectors.

\section{MATERIALS AND METHODS}

\section{Cells and viruses}

Established lepidopteran cell lines $S$. frugiperda IPLB-SF21 (SF21) (16) and T. $n i$ TN368 (17) were propagated in TC100 growth medium (GIBCO Laboratories) supplemented with fetal bovine serum and $2.6 \mathrm{mg}$ of tryptose broth per $\mathrm{mL}$ as described (4). Viruses used included the wild-type L-1 strain of AcMNPV (18), an AcMNPV polyhedrin-deletion mutant (kindly provided by L. K. Miller, University of Georgia), and AcMNPV-deletion mutants $v \Delta 35 \mathrm{~K}$ and $\mathrm{v} \Delta 35 \mathrm{~K} / \mathrm{lacZ}$ that lack the $p 35$ promoter and 287 (of 299) codons of the $p 35$ open reading frame as a result of the deletion of a 950-bp NruI-SpeI fragment within the EcoRI-S region (8). For infection, cells were inoculated with extracellular $\mathrm{BV}$ and rocked for $1 \mathrm{~h}$ at room temp, after which the residual inoculum was removed and replaced with growth medium. When indicated, G418 (Geneticin, GIBCO Laboratories) was added to the growth medium.

\section{Transfer plasmids}

To generate neo-containing transfer plasmids, neo was first placed under control of the promoter for the AcMNPV p35 gene. To this end, a 1.0-kb $B g l \mathrm{II}-\mathrm{SmaI}$ fragment from plasmid pSV2-neo (10) was inserted into the BamHI and SpeI (end repaired with the Klenow fragment) sites, respectively, of plasmid p35KPRM (19) that contains nucleotides -226 to +12 of the $p 35$ promoter (RNA start site, +1 ). Next, the $p 35$ promoter-neo cassette was inserted as a 1.3-kb XhoI-XbaI fragment into the corresponding sites of transfer vector $\mathrm{pEVocc}+\mathrm{PA}$ (5) to generate plasmid $\mathrm{pEVocc}^{+} /$neo. Transfer plasmid $\mathrm{pEV}$-lacZ/neo was constructed by inserting a 3.3-kb XbaI-KpnI fragment (end repaired at the $X b a I$ site), containing the Escherichia coli lacZ gene under control of the polyhedrin promoter, into XhoI (end repaired) and $K p n I$ sites of $\mathrm{pEVocc}+/$ neo; this replaced the polyhedrin gene with $l a c Z$. The $X b a I-K p n I ~ l a c Z$ fragment was obtained from plasmid pPolylacZ in which the polyhedrin promoter was fused to the lacZ gene in the pBluescript (KS) vector (Stratagene). Transfer plasmid $\mathrm{pEVocc}+/ 35 \mathrm{~K}^{+}$was constructed by inserting a $1.3-\mathrm{kb}$ $X h o I-X b a I$ fragment, containing the complete $p 35$ gene [including open reading frame and promoter from plasmid p35K-ORF (8)], into the corresponding sites of $\mathrm{pEV} / \mathrm{occ}^{+} / \mathrm{PA}$. The transfer plasmid $\mathrm{pEV}$-lacZ/35K $\mathrm{K}^{+}$was constructed by inserting the 3.3-kb lacZ-containing $\mathrm{XbaI}$-KpnI fragment (end repaired at the

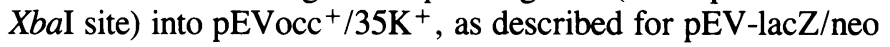
(see above).

\section{Generation of AcMNPV recombinants}

SF21 cultures $\left(2 \times 10^{6}\right.$ cells) were transfected with viral DNA $(1 \mu \mathrm{g})$ and linearized transfer plasmid $(10 \mu \mathrm{g})$ using Lipofectin (Bethesda Research Laboratories) as previously described (19). When transfections were conducted using whole virus, Lipofectin $(30 \mu \mathrm{g})$ was added directly to a mixture $(50 \mu \mathrm{L})$ of growth medium containing approximately $6 \times 10^{5} \mathrm{PFU}$ of virus mutant $\mathrm{v} \Delta 35 \mathrm{~K}$ and the transfer plasmid. After a $15 \mathrm{~min}$ incubation at room temp, the virus-plasmid mixture was added dropwise to SF21 monolayers. Recombinant viruses were harvested 4 days posttransfection, plaque purified using SF21 cells, and characterized by restriction mapping of genomic DNA (data not shown). Linearized viral DNA was used essentially as described previously (20). In brief, $200 \mathrm{ng}$ of $\mathrm{v} \Delta 35 \mathrm{~K} / \mathrm{lacZ}$ DNA was digested with Bsu36I (New England Biolabs), mixed with $2 \mu \mathrm{g}$ of plasmid $\mathrm{pEVocc}+35 \mathrm{~K}^{+}$and Lipofectin, then added to $2 \times 10^{6} \mathrm{SF} 21$ cells. Virus was harvested 2 days posttransfection. Four recombinant viruses (Fig. 1) were generated with the indicated transfer plasmids and parental virus DNAs: 1) vNeo was selected by its occlusion-positive $\left(\mathrm{occ}^{+}\right)$plaque phenotype after transfection with $\mathrm{pEVocc}+/$ neo and an AcMNPV polyhedrin-deletion mutant, 2) vNeo/lacZ was selected by its blue, occlusion-negative $\left(\mathrm{occ}^{-}\right)$phenotype in the presence of $\mathrm{X}$ Gal (see below) after transfection with pEV-lacZ/neo and wildtype AcMNPV, 3) v $\Delta 35 \mathrm{~K} / 35 \mathrm{~K}^{+}$was selected by its occ ${ }^{+}$ phenotype after transfection with $\mathrm{pEVocc}+/ 35 \mathrm{~K}^{+}$and $\mathrm{v} \Delta 35 \mathrm{~K} / \mathrm{lacZ}$, and 4) $\mathrm{v} \Delta 35 \mathrm{~K} / \mathrm{lacZ} / 35 \mathrm{~K}^{+}$was selected by its blue, $\mathrm{occ}^{-}$phenotype after transfection with $\mathrm{pEV}$-lacZ/35K $\mathrm{K}^{+}$and $\mathrm{v} \Delta 35 \mathrm{~K}$. Viruses $\mathrm{vNeO}$ and $\mathrm{vNeo} / \mathrm{lacZ}$ were identified and isolated in the absence of G418 to avoid neo selection.

\section{Determination of virus titers}

When wild-type and neo-containing viruses were compared, titers were determined by using SF21 cells in standard plaque assays 
(4). Due to differences in plaquing efficiency on SF21 cells, titers of both $p 35$-deletion mutants and p35-containing viruses were determined using TN368 cells; multiplication of AcMNPV recombinants with or without $p 35$ is indistinguishable in TN368 cells (8). When indicated, expression of $\beta$-galactosidase by lacZcontaining viruses was visualized by including $100 \mu \mathrm{g}$ of X-Gal (5-bromo-4-chloro-3-indolyl- $\beta$-D-galactopyranoside) per $\mathrm{mL}$ in the agarose overlay. When multiple phenotypes were present on the same plate, plaques were distinguished by using a dissecting microscope.

\section{RESULTS}

Neo provides a growth advantage to AcMNPV recombinants in the presence of $\mathbf{G 4 1 8}$

To test the effectiveness of neo as a positive selector for AcMNPV, we constructed two recombinant viruses in which neo was inserted at the nonessential polyhedrin locus (Fig. 1B), either adjacent to the polyhedrin gene itself (vNeo) or a lac $\bar{Z}$ reporter gene fused to the polyhedrin promoter ( $\mathrm{vNeO} / \mathrm{lacZ}$ ). In both cases, neo was placed under control of the AcMNPV p35 promoter that has both early and late regulatory elements $(19,21)$. The effect of G418 on multiplication of neo-containing viruses was then compared to that of wild-type virus by inoculating SF21 cultures at a multiplicity of infection (MOI) of 0.05 plaque-forming units (PFU) per cell. This low MOI was used to minimize the frequency with which cells were infected with more than one virus, a requirement for selective amplification of these viruses (see below). The yield of BV accumulated over a 48-h period from cultures treated with G418 was determined by plaque assay. Increasing concentrations of drug resulted in a progressive reduction in the yield of wild-type virus (Fig. 2). At the highest concentrations of G418, wild-type BV yields were reduced more than 1,700-fold. In contrast, BV yields of neo-containing viruses,

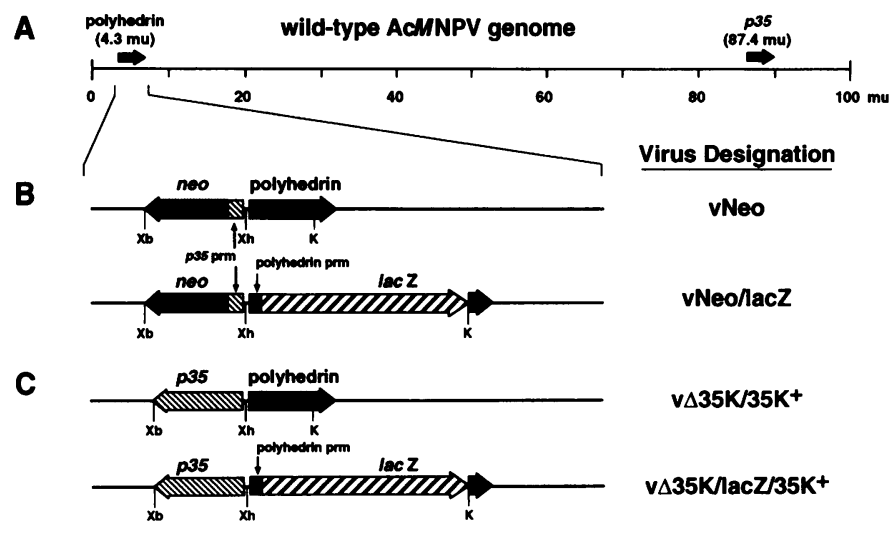

Figure 1. Genome organization of AcMNPV recombinants. (A) Position of the polyhedrin gene [4.3 map units (mu)] and p35 $(87.4 \mathrm{mu})$ on a linear map of the wild-type AcMNPV genome. The gene organization of the polyhedrin locus is illustrated for (B) the neo-containing viruses, vNeo and vNeo/lacZ, and (C) the $p 35$-containing viruses, $v \Delta 35 \mathrm{~K} / 35 \mathrm{~K}^{+}$and $v \Delta 35 \mathrm{~K} / \mathrm{lacZ} / 35 \mathrm{~K}^{+}$. The lacZ gene replaced the polyhedrin gene within viruses $v N e o / l a c Z$ and $v \Delta 35 \mathrm{~K} / \mathrm{lacZ} / 35 \mathrm{~K}^{+}$, while $p 35$ was removed from its native position within the genome of viruses $\mathrm{v} \Delta 35 \mathrm{~K} / \mathrm{lacZ} / 35 \mathrm{~K}^{+}$and $\mathrm{v} \Delta 35 \mathrm{~K} / 35 \mathrm{~K}^{+}$by deletion of a 950 -bp $N r u \mathrm{I}$-Spel fragment $(87.0-87.7 \mathrm{mu})$. Shaded arrows depict the promoter (prm), coding region, and direction of transcription of each gene. The $p 35$ and polyhedrin genes were controlled by their wild-type promoter. Restriction site abbreviations: K, KpnI, $\mathrm{Xb}, \mathrm{XbaI}, \mathrm{Xh}, \mathrm{XhoI}$.
$\mathrm{vNeo}$ and $\mathrm{vNeo} / \mathrm{lacZ}$, were reduced only 2 - to 5 -fold compared to that in the absence of drug (Fig. 2). This low level of inhibition may be due in part to an observed cytotoxicity of G418 $(2.5 \mathrm{mg} / \mathrm{mL})$ in both infected and uninfected cells. Because of its lack of cytotoxicity during infection and the 1,200-fold reduction in wild-type virus yields (Fig. 2), G418 was used at a concentration of $2.0 \mathrm{mg} / \mathrm{mL}$ throughout this study. In other experiments, we have noted some variation in the overall level of inhibition of wild-type virus growth which is probably due to differences in commercial G418 preparations (22).

Differential inhibition of AcMNPV-specific protein synthesis by $\mathbf{G 4 1 8}$

To examine the mechanism by which $\mathrm{G} 418$ interfered with AcMNPV replication, we compared levels of protein synthesis in virus-infected cells treated with G418. Analysis by pulselabeling with $\left[{ }^{35} \mathrm{~S}\right]$ methionine/cysteine indicated that the drug reduced the rate of protein synthesis in cells infected with wildtype virus, but not the neo-containing virus vNeo (Fig. 3). By $7 \mathrm{~h}$ after infection, there was no discernable difference in the rate or pattern of protein synthesis in the presence or absence of G418. However, by $24 \mathrm{~h}$, the synthesis of several prominent virus proteins (including gp64 and polyhedrin) was reduced in cells infected with wild-type virus (lane 6), even though the overall rate of protein synthesis of treated and untreated cultures was comparable. Other virus-specific proteins (see dots) were not affected to the same extent. A similar pattern of selective inhibition by $\mathrm{G} 418$ was observed in cultured mammalian cells infected with vaccinia virus (11). By $48 \mathrm{~h}$ after infection, G418 dramatically reduced the rate of synthesis of most proteins in wildtype AcMNPV-infected cells (lane 10). In contrast, the drug had no apparent effect on the levels or timing of proteins synthesized in vNeo-infected cells. Thus, expression of neomycin-resistance circumvented the inhibition of virus protein synthesis by G418.

\section{Selective amplification of neo-containing viruses}

To test whether the resistance to G418 conferred by neo was sufficient to selectively amplify AcMNPV recombinants in mixtures where such viruses were in the minority, we monitored

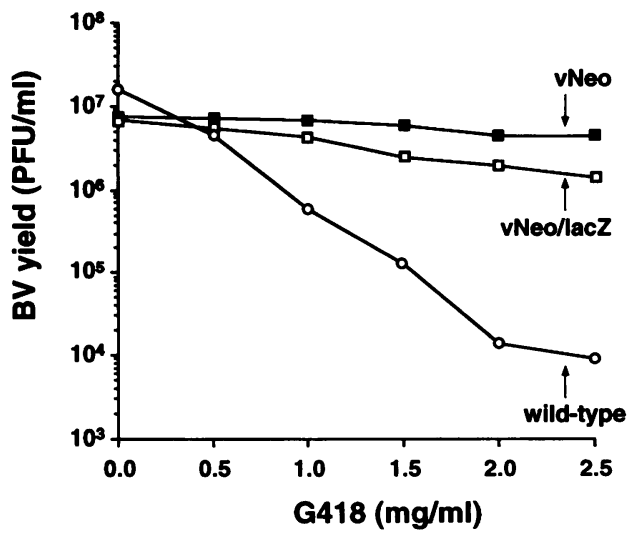

Figure 2. Effects of $\mathrm{G} 418$ on production of $\mathrm{BV}$ from wild-type and neo-containing viruses. SF21 cultures $\left(2 \times 10^{6}\right.$ cells) were inoculated with wild-type, vNeo, and vNeo/lacZ viruses $(0.05 \mathrm{PFU} / \mathrm{cell})$ and covered with growth medium containing the indicated concentration of G418. The medium was collected $48 \mathrm{~h}$ after infection and the virus titer was determined by plaque assay using SF21 cells. The yield of progeny $\mathrm{BV}(\mathrm{PFU} / \mathrm{mL})$ is indicated using a $\log$ scale. 


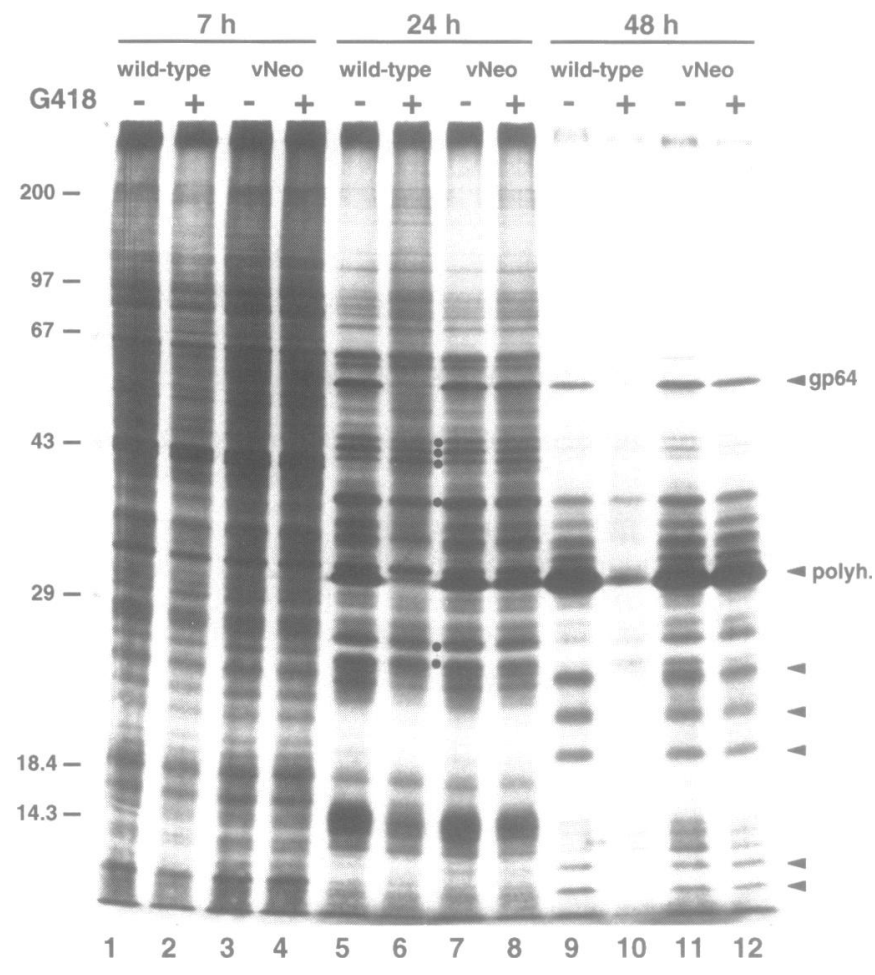

Figure 3. Effects of G418 on protein synthesis in vNeo and wild-type virus infected cells. SF21 cells were inoculated with either wild-type or vNeo viruses (10 $\mathrm{PFU} /$ cell) and covered with growth medium with $(+)$ or without (-) G418 $(2 \mathrm{mg} / \mathrm{mL})$. At 6,23 , and $47 \mathrm{~h}$ after infection, cells were radiolabeled for $1 \mathrm{~h}$ with $\left[{ }^{35}\right.$ S]methionine-cysteine, lysed, and analyzed by SDS-polyacrylamide gel electrophoresis $(23,24)$. The positions of molecular weight standards (size in kilodaltons) are shown to the left of the fluorogram. Radiolabeled proteins that were unaffected (dots) or affected (triangles) by G418 are indicated, including polyhedrin (polyh) and the major BV glycoprotein, gp64

virus multiplication during serial passage of wild-type and neocontaining viruses under G418 selection. SF21 cultures were inoculated with experimental mixtures of wild-type virus and $\mathrm{vNeo} / \mathrm{lacZ}$ using ratios of $10^{3}, 10^{4}$, and $10^{5}$ to 1 , respectively. To minimize helper virus activity, expected to occur when both viruses infect the same cell, the first infection was initiated at a low MOI (0.05 PFU/cell); subsequent passages represented blind (unknown MOI) inoculations using growth medium from the previous infection. The level of each virus was determined by plaque assay in the presence of $\mathrm{X}-\mathrm{Gal}$ to distinguish $\mathrm{vNeo} / \mathrm{lacZ}$ (blue, $\mathrm{occ}^{-}$plaques) from wild-type virus (colorless, $\mathrm{occ}^{+}$ plaques). Over four serial passages, vNeo/lacZ increased progressively (Fig. 4). The final ratio of wild-type virus to $\mathrm{vNeo} / \mathrm{lacZ}$ for the starting mixtures $\left(10^{3}, 10^{4}\right.$, and $10^{5}$ to 1$)$ was 7,81 , and 200 to 1 , respectively. Thus, the greatest overall increase in $\mathrm{vNeo} / \mathrm{lacZ}(4,200$-fold) occurred within the virus mixture having the lowest starting ratio, $10^{5}$ to 1 (Fig. $4 \mathrm{C}$ ). In general, four serial passages resulted in a 100 - to 500 -fold increase in the proportion of neo-containing virus. In contrast to the neo-containing virus, the level of wild-type virus remained steady after a small initial increase in all mixtures. As shown in Fig. 2, G418 reduces the yield of wild-type virus more than 1000 -fold, but fails to block virus growth completely. Thus, the nearly constant levels of wild-type virus during serial passage suggested that the yield of wild-type progeny was about the same as that used for inoculum. This was supported by other
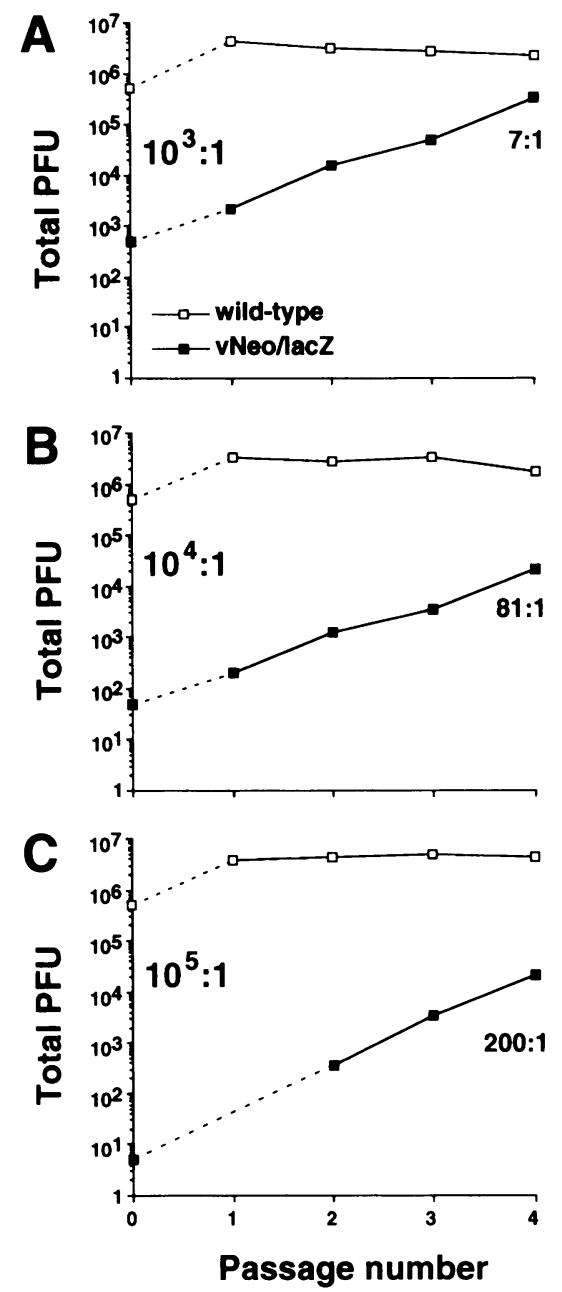

Figure 4. Selective amplification of neo-containing recombinant $\mathrm{vNeo} / \mathrm{lacZ}$ by serial passage. SF21 cultures $\left(10^{7}\right.$ cells) were inoculated at a constant MOI $(0.05$ PFU/cell) with the indicated mixtures A) $10^{3}: 1$, B) $10^{4}: 1$, and C) $10^{5}: 1$ of wildtype and $v N e o / l a c Z$ viruses, respectively, and covered with growth medium $(5 \mathrm{~mL})$ containing G418 (2 mg/mL). The medium was collected $30 \mathrm{~h}$ after infection and $4 \mathrm{~mL}$ was used to inoculate a subsequent culture $\left(10^{7}\right.$ cells). The virus titer of the remaining medium ( $1 \mathrm{~mL}$ ) was determined by plaque assay using SF21 cells and X-Gal. The yield (Total PFU) of wild-type and vNeo/lacZ viruses is shown for each the four successive infections using a log scale. The initial level of input virus is indicated on the $\mathrm{Y}$ axis and the determined ratio of wild-type to $\mathrm{vNeo} / \mathrm{lacZ}$ virus for the last passage is indicated on the right.

Table 1. BV yields of AcMNPV recombinants with or without $p 35^{a}$

\begin{tabular}{llc}
\hline Virus & $\begin{array}{l}\text { BV production } \\
\text { (total PFU) }\end{array}$ & $\begin{array}{l}\text { Fold increase } \\
\text { over } v 335 \mathrm{~K}\end{array}$ \\
\hline wild-type & $1.2 \pm 0.31 \times 10^{7}$ & 1000 \\
$\mathrm{v} \Delta 35 \mathrm{~K}$ & $1.2 \pm 0.26 \times 10^{4}$ & 1 \\
$\mathrm{v} \Delta 35 \mathrm{~K} / 35 \mathrm{~K}^{+}$ & $4.4 \pm 0.56 \times 10^{6}$ & 370 \\
$\mathrm{v} \Delta 35 \mathrm{~K} / \mathrm{lacZ} / 35 \mathrm{~K}^{+}$ & $1.4 \pm 0.26 \times 10^{7}$ & 1200
\end{tabular}

a SF21 cultures $\left(2 \times 10^{6}\right.$ cells $)$ were infected with the indicated viruses at 0.05 PFU per cell. The yield of budded virus (BV) produced after $48 \mathrm{~h}$ was determined by plaque assay using TN368 cells. Values shown represent the average of triplicate infections.

experiments in which the level of wild-type virus produced by a single, low MOI inoculation (0.05 PFU/cell) of G418-treated cells was similar to the input virus (data not shown). 

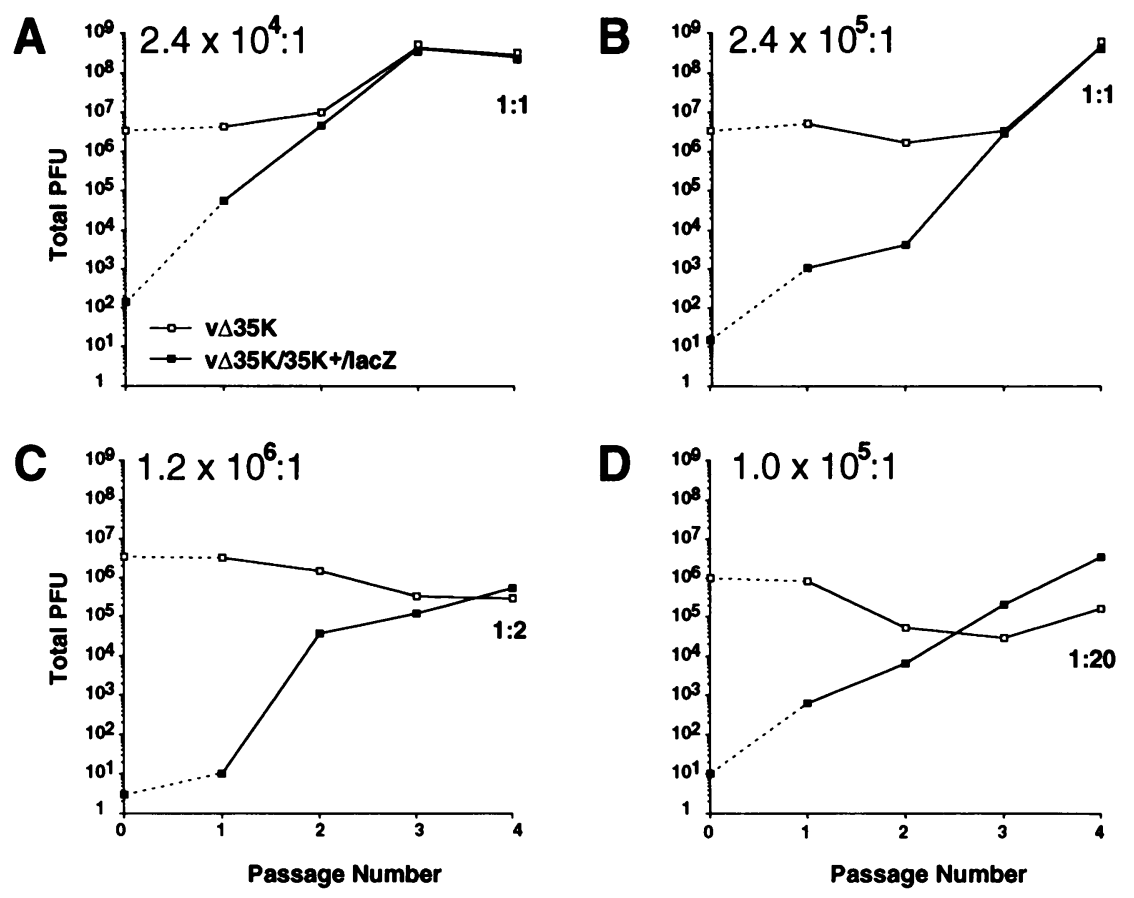

Figure 5. Selective amplification of the $p 35$-containing virus $v \Delta 35 \mathrm{~K} / \mathrm{lacZ} / 35 \mathrm{~K}^{+}$by serial passage. Replicate $\mathrm{SF} 21$ cultures $\left(5 \times 10^{6}\right.$ cells/plate $)$ were inoculated with the indicated mixtures A) $2.4 \times 10^{4}: 1$, B) $2.4 \times 10^{5}: 1$, C) $1.2 \times 10^{6}: 1$, and D) $10^{5}: 1$ of viruses $v \Delta 35 \mathrm{~K}$ and v $\Delta 35 \mathrm{~K} /$ lacZ/35K $\mathrm{K}^{+}$, respectively. Cells were inoculated at an MOI of $0.7 \mathrm{PFU} /$ cell (A, B, and C) or $0.1 \mathrm{PFU} /$ cell (D) using virus stocks with titers determined by plaque assay using TN368 cells. After a 1-h adsorption, the inoculum was removed and replaced with growth medium $(5 \mathrm{~mL})$. All of the medium was collected $48 \mathrm{~h}$ after infection and used to inoculate the next culture. The level of progeny viruses was determined in a parallel infection by plaque assays using TN368 cells and X-Gal. The initial level of input viruses is indicated on the $\mathrm{Y}$ axis (log scale) and virus yields (Total PFU) are shown for four passages. The determined ratio of $v \Delta 35 \mathrm{~K}$ to $\mathrm{v} \Delta 35 \mathrm{~K} / \mathrm{lacZ} / 35 \mathrm{~K}^{+}$for the last passage is indicated on the right.

Table 2. Proportion of viruses generated by transfection with transfer plasmid $\mathrm{pEV}$-lacZ/35K $\mathrm{K}^{+}$and parental virus $\mathrm{v} \Delta 35 \mathrm{~K}$

\begin{tabular}{|c|c|c|c|}
\hline \multirow[t]{2}{*}{ Virus } & \multirow{2}{*}{$\begin{array}{l}\text { SF21 plaque } \\
\text { phenotype }\end{array}$} & \multicolumn{2}{|c|}{ Fraction of Total Plaques $(\%)^{a}$} \\
\hline & & Purified DNA ${ }^{b}$ & Intact Virus \\
\hline $\begin{array}{l}\mathrm{v} \Delta 35 \mathrm{~K} / \mathrm{lacZ} / 35 \mathrm{~K}^{+} \\
\text {(desired) }\end{array}$ & blue, occ $^{-}$ & 15 & 30 \\
\hline $\begin{array}{l}\mathrm{v} \Delta 35 \mathrm{~K} \\
\text { (parental) }\end{array}$ & colorless, $\mathrm{occ}^{-}$ & 41 & 35 \\
\hline $\begin{array}{l}\operatorname{lac} \mathrm{Z}^{+}, 35 \mathrm{~K}^{+} \\
\text {(other) }\end{array}$ & blue, occ $^{+}$ & 20 & 10 \\
\hline $\begin{array}{l}35 \mathrm{~K}^{+} \\
\text {(other) }\end{array}$ & colorless, occ $^{+}$ & 24 & 25 \\
\hline
\end{tabular}

${ }^{a}$ Virus was harvested 4 days postransfection and screened by plaque assay using SF21 cells in the presence of X-Gal; occlusion-negative $\left(\mathrm{occ}^{-}\right)$, occlusion-positive $\left(\mathrm{occ}^{+}\right)$. Values shown represent the average of two independent transfections which did not vary more than $\pm 3 \%$.

${ }^{b}$ Purified, circular v $\Delta 35 \mathrm{~K}$ DNA $(1 \mu \mathrm{g})$ was transfected with pEV-lacZ/35K ${ }^{+}(10 \mu \mathrm{g})$ using $2 \times 10^{6} \mathrm{SF} 21$ cells.

${ }^{c}$ An equal volume of $v \Delta 35 \mathrm{~K}$ BV $\left(6 \times 10^{5} \mathrm{PFU}\right)$ was substituted for purified viral DNA.

p35 confers a growth advantage to AcMNPV recombinants p35-deletion viruses cause premature cell lysis of SF21 cultures and produce low yields of BV $(6,8)$. To test the effectiveness of $p 35$ as a selectable marker, we examined the growth properties and selectability of AcMNPV recombinants (Fig. 1C) in which a copy of $p 35$ under control of its own promoter was inserted at the polyhedrin locus of $p 35$-deletion mutant $v \Delta 35 \mathrm{~K}$. When p35 was inserted either adjacent to the polyhedrin gene itself $\left(\mathrm{v} \Delta 35 \mathrm{~K} / 35 \mathrm{~K}^{+}\right)$or adjacent to the lacZ reporter gene fused to the polyhedrin promoter $\left(\mathrm{v} \Delta 35 \mathrm{~K} / \mathrm{lacZ} / 35 \mathrm{~K}^{+}\right)$, the accumulated yield of BV over a 48-h period was 370- and 1200 -fold higher, respectively, than that of $\mathrm{v} \Delta 35 \mathrm{~K}$ (Table 1). Using low MOI (0.05 PFU/cell) inoculations, the accumulated BV yield of $v \Delta 35 \mathrm{~K}$ was 1000 -fold lower than that of wild-type virus. Thus, insertion of $p 35$ restored growth of $p 35$-deletion mutant to levels similar to that of wild-type virus. Other studies have indicated that growth differences between viruses that contain or lack $p 35$ vary with the MOI (8). 


\section{Selective amplification of $p 35$-containing viruses}

To assess the selectability conferred by $p 35$, we monitored virus multiplication during serial passage of mixtures of deletion mutant $\mathrm{v} \Delta 35 \mathrm{~K}$ and $\mathrm{v} \Delta 35 \mathrm{~K} / \mathrm{lacZ} / 35 \mathrm{~K}^{+}$, where the later virus was in the minority. SF21 cultures were inoculated at an MOI of $0.7 \mathrm{PFU} /$ cell and the yield of each virus during serial passage was determined. Plaque assays were conducted using TN368 cells and $\mathrm{X}-\mathrm{Gal}$ to distinguish $\mathrm{v} \Delta 35 \mathrm{~K}$ (colorless, $\mathrm{occ}^{+}$plaques) from $\mathrm{v} \Delta 35 \mathrm{~K} / \mathrm{lacZ} / 35 \mathrm{~K}^{+}$(blue, occ ${ }^{-}$plaques). For each of the virus mixtures, the level of $p 35$-containing virus $v \Delta 35 \mathrm{~K} / \mathrm{lacZ} / 35 \mathrm{~K}^{+}$ increased dramatically (Figs. 5A-5C). After four consecutive passages, the ratio of $v \Delta 35 \mathrm{~K}$ to $\mathrm{v} \Delta 35 \mathrm{~K} / \mathrm{lacZ} / 35 \mathrm{~K}^{+}$was 1,1 , and 0.5 to 1 for the starting ratios of $2.4 \times 10^{4}, 2.4 \times 10^{5}$, and $1.2 \times 10^{6}$ to 1 , respectively. This corresponded to 24,000 -fold, 240,000 -fold, and $2.4 \times 10^{6}$-fold increases in the proportion of p35-containing virus.

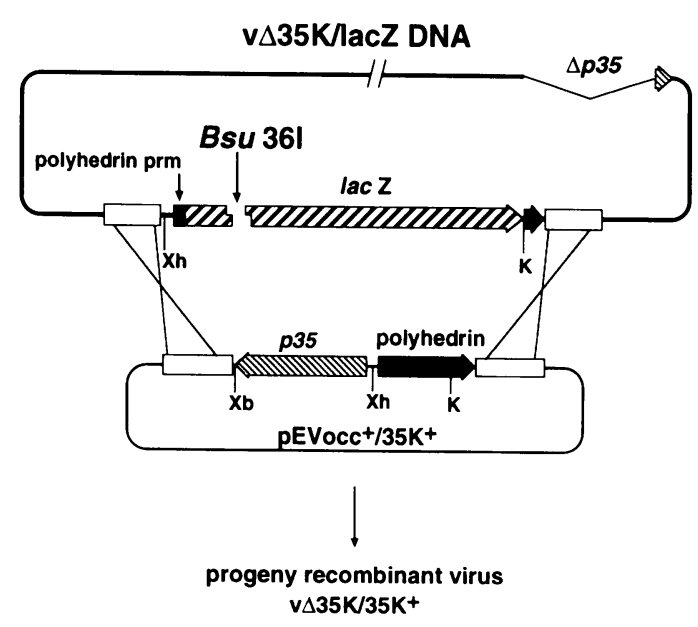

Figure 6. Strategy for gene replacement using linear $v \Delta 35 \mathrm{~K} / \mathrm{lacZ}$ virus DNA. Homologous recombination between $v \Delta 35 \mathrm{~K} / \mathrm{lacZ}$ virus DNA (top) and transfer plasmid $\mathrm{pEVocc}{ }^{+} / 35 \mathrm{~K}^{+}$(bottom) replaced the viral lacZ gene with a functional polyhedrin gene. The resulting recombinant virus $\left(\mathrm{v} \Delta 35 \mathrm{~K} / 35 \mathrm{~K}^{+}\right)$exhibited an occlusion-positive $\left(\mathrm{occ}^{+}\right.$) plaque phenotype. $\mathrm{v} \Delta 35 \mathrm{~K} / \mathrm{lacZ} \mathrm{DNA}$ was digested with $B s u 36$ that linearized the genome at a unique site within the lac $Z$ gene. Shaded arrows depict the promoter (prm), coding region, and direction of transcription of each gene; the $p 35$ and polyhedrin genes were controlled by their wild-type promoter. DNA sequences (open boxes) for homologous recombination between the virus and transfer plasmid are located on either side of the polyhedrin locus. Restriction site abbreviations are listed in the legend to Figure 1.
During later passages when the levels of viruses $\mathrm{v} \Delta 35 \mathrm{~K} / \mathrm{lacZ} / 35 \mathrm{~K}^{+}$and $\mathrm{v} \Delta 35 \mathrm{~K}$ were high and subsequent MOIs were boosted proportionately, both viruses increased in parallel (Fig. 5A and 5B). This suggested that $v \Delta 35 \mathrm{~K} / \mathrm{lacZ} / 35 \mathrm{~K}^{+}$ provided helper virus activity for $\mathrm{v} \Delta 35 \mathrm{~K}$. To reduce such activity, we initiated a serial passage at a lower MOI $(0.1 \mathrm{PFU} /$ cell $)$ using an initial $v \Delta 35 \mathrm{~K}$ to $v \Delta 35 \mathrm{~K} / \mathrm{lacZ} / 35 \mathrm{~K}^{+}$ratio of $10^{5}$ to 1 . After four passages (Fig. 5D), a higher fraction of $v \Delta 35 \mathrm{~K} / \mathrm{lacZ} / 35 \mathrm{~K}^{+}$ (20 to 1) was obtained, even though the overall increase ( $2 \times 10^{6}$-fold) of this virus was identical to that obtained with a higher MOI (Fig. 5C). Thus, by maintaining a low MOI through consecutive passages, the proportion of $p 35$-containing virus would be expected to increase even further and also minimize the potential for defective virus formation.

\section{Use of $p 35$ as a selectable marker in the generation of} AcMNPV recombinants

The relative strength of selectivity conferred by $p 35$ suggested that it would expedite the recovery of recombinant viruses with new (foreign) DNA inserted into the viral genome by allelic replacement, a method that typically yields desired recombinants at a frequency of $0.1 \%$ to $1 \%$ of the total progeny virus (4). To test this possibility, we transfected SF21 cells with deletion mutant $\mathrm{v} \Delta 35 \mathrm{~K}$ and a polyhedrin-based transfer plasmid (pEVlacZ $/ 35 \mathrm{~K}^{+}$) that contains $p 35$ and its promoter immediately adjacent to the $l a c Z$ reporter gene under control of the polyhedrin promoter. The proportion of desired recombinants containing lac $Z$ and $p 35$ inserted at the polyhedrin locus (resulting in loss of the polyhedrin gene) was determined. Since acquisition of $p 35$ restores polyhedrin promoter-mediated gene expression (8), the desired $\mathrm{v} \Delta 35 \mathrm{~K} / \mathrm{lacZ} / 35 \mathrm{~K}^{+}$recombinants (blue, occ ${ }^{-}$plaques) were distinguished from parental $\mathrm{v} \Delta 35 \mathrm{~K}$ (colorless, $\mathrm{occ}^{-}$ plaques) by plaque assay using SF21 cells. When purified $\mathrm{v} \Delta 35 \mathrm{~K}$ DNA was used, desired recombinants comprised $15 \%$ of the transfection progeny, compared to parental virus that comprised $41 \%$ (Table 2). To simplify transfections, viral DNA was replaced with intact $v \Delta 35 \mathrm{~K} B V$ by using an equal volume of $\mathrm{v} \Delta 35 \mathrm{~K}$-containing growth medium. When intact virus was used, the proportion of desired recombinants increased to $30 \%$ of the total progeny (Table 2).

While more than $60 \%$ of the transfection progeny exhibited plaque phenotypes consistent with acquisition of $p 35$, the phenotype of more than half of these recombinant viruses (colorless, $\mathrm{Occ}^{+}$and blue, $\mathrm{occ}^{+}$plaques) indicated that insertion occurred at sites other than the polyhedrin locus (Table 2). Southern blot analysis of viral DNA from representative colorless,

Table 3. Proportion of AcMNPV recombinants using linearized and circular DNA from $v \Delta 35 \mathrm{~K} / \mathrm{lacZ}{ }^{a}$

\begin{tabular}{|c|c|c|c|c|c|c|c|c|}
\hline \multirow[t]{4}{*}{ Virus DNA } & \multicolumn{8}{|c|}{ Fraction of Total Plaques (\%) } \\
\hline & \multicolumn{4}{|c|}{ SF21 } & \multicolumn{4}{|c|}{ TN368 } \\
\hline & \multicolumn{2}{|c|}{ Desired $^{b}$} & \multicolumn{2}{|c|}{ Other ${ }^{c}$} & \multicolumn{2}{|c|}{ Desired $^{b}$} & \multicolumn{2}{|c|}{ Other $c$} \\
\hline & A. & B. & A. & B. & A. & B. & A. & B. \\
\hline Circular & 97 & 17 & 24 & 83 & 76 & 3 & 3 & 97 \\
\hline Linearized & 96 & 87 & 4 & 13 & 92 & 82 & 8 & 18 \\
\hline
\end{tabular}


occ $^{+}$plaques confirmed the insertion of $p 35$ at alternate genomic sites (data not shown). Thus, these undesired recombinants were probably generated through low-level nonhomologous recombination between the transfer plasmid and parental $\mathrm{v} \Delta 35 \mathrm{~K}$ that were selectively amplified due to acquisition of $p 35$. In contrast, upon replating on SF21 cells, $90 \%$ of the blue, $\mathrm{occ}^{+}$plaques generated multiple plaque phenotypes (data not shown). This suggested that most of the blue, $\mathrm{occ}^{+}$plaques represented mixed infections in which polyhedrin and lacZ were encoded by separate viruses.

\section{Linearization of viral DNA facilitates site-specific insertion} of the p35 selectable marker

To direct recombination to a single site and thus reduce the fraction of recombinants in which the $p 35$ marker inserted at undesired locations, we determined the effect of linearizing parental virus DNA prior to transfection. It has been shown previously that plasmid recombination across the endonucleasegenerated gap of linearized viral DNA produces a higher fractional yield (up to $30 \%$ ) of recombinant viruses, most likely through the recircularization of DNA that is required for infectivity (20). To this end, DNA from deletion mutant $\mathrm{v} \Delta 35 \mathrm{~K} / \mathrm{lacZ}$ was linearized with $B s u 36 \mathrm{I}$ at a unique site within the lacZ gene and transfected with transfer plasmid $\mathrm{pEVocc}^{+} / 35 \mathrm{~K}^{+}$. Plasmid recombination across the resulting gap was predicted to generate a virus $\left(\mathrm{v} \Delta 35 \mathrm{~K} / 35 \mathrm{~K}^{+}\right)$in which the lac $Z$ gene of parent $v \Delta 35 \mathrm{~K} / \mathrm{lacZ}$ was replaced by the polyhedrin and $p 35$ genes (Fig. 6).

Compared to circular (mock-digested) DNA, linearized virus DNA yielded a higher proportion of desired $v \Delta 35 \mathrm{~K} / 35 \mathrm{~K}^{+}$ viruses (Table 3). When plated on SF21 cells, up to $96 \%$ of the progeny was comprised of desired $p 35$-containing recombinants; these viruses produced colorless, occ $^{+}$plaques that were distinguished from the occ ${ }^{-}$plaques of parent $v \Delta 35 \mathrm{~K} / \mathrm{lacZ}$. Restriction mapping of twelve randomly picked viruses with a colorless, $\mathrm{occ}^{+}$phenotype demonstrated that each had the predicted genome structure for $\mathrm{v} \Delta 35 \mathrm{~K} / 35 \mathrm{~K}^{+}$(data not shown). The high proportion of desired recombinants was confirmed by plaque assay using TN368 cells in which the desired (colorless, $\mathrm{occ}^{+}$) and parental (blue, $\mathrm{occ}^{-}$) viruses have similar plaquing efficiencies. By this analysis, linearized DNA increased the proportion of desired viruses from $3 \%$ to about $87 \%$ (Table 3). When linearized $v \Delta 35 \mathrm{~K} / \mathrm{lacZ}$ DNA was transfected with a transfer plasmid that contained a defective copy of p35 $\left(\mathrm{pEVocc}^{+} / 35 \mathrm{~K}^{\Delta \mathrm{Spe}}\right)(8)$, no desired recombinant viruses were detected using TN368 cells (data not shown). In contrast, transfections with circular $v \Delta 35 \mathrm{~K} / \mathrm{lacZ}$ DNA and the same transfer plasmid yielded approximately $1 \%$ desired recombinants. This finding demonstrated that a functional copy of $p 35$ was required for the high proportion of desired recombinant viruses, and that linearization of parental DNA alone was not responsible.

\section{DISCUSSION}

In this report, we have identified two genes, AcMNPV-encoded p35 and neo, that confer dominant selectivity to baculoviruses upon insertion into the viral genome. Use of these positive genetic markers makes it possible to selectively amplify recombinant (or mutant) viruses amid a high background of parental virus. Thus, by facilitating the generation of virus mutants, these genes will be useful for continued studies on baculovirus gene function and organization. The especially strong selectivity provided by $p 35$ also facilitates the construction and isolation of AcMNPV expression vectors.

\section{Neo as a selectable marker for AcMNPV recombinants}

When added at the time of inoculation, G418 reduced the accumulated yield of wild-type BV greater than 1,000-fold and thus demonstrated that this antibiotic is an effective inhibitor of AcMNPV multiplication (Fig. 2). Our preliminary results suggested that G418 inhibits virus growth by reducing the synthesis of selected late viral proteins. In contrast, when neo was placed under control of the promoter for $p 35$, late virus protein synthesis and $\mathrm{BV}$ production was restored; $\mathrm{BV}$ yields were only 2- to 5-fold lower than that in the absence of drug (Fig. 2). Thus, virus-mediated expression of neo was capable of conferring G418 resistance. As demonstrated by serial passage of virus mixtures in the presence of G418, neo also conferred dominant selectivity to AcMNPV recombinants. As high as a 500-fold increase in the neo-containing recombinant vNeo/lacZ (relative to wild-type virus) was obtained over four consecutive passages (Fig. 4). We have not yet tested whether a different AcMNPV promoter would improve virus selectivity. The strong p10 promoter of Galleria mellonella nuclear polyhedrosis virus has been used to direct neo expression in infected Lymantria dispar cells (25). Although recovery of neo-containing virus required a prolonged period of G418 selection (10 serial passages of 6 days each), this indicated that neo can be used for selection of other baculoviruses. Neo selectivity may therefore be useful in studies that monitor the movement of marked transposons between different baculoviruses and their invertebrate hosts (see below).

$\mathrm{Neo}$ is the first drug resistance marker for positive selection of baculoviruses. Recently, the herpes simplex virus type 1 thymidine kinase gene (HSV1-tk) was shown to be an effective marker for negative selection of baculoviruses (26). Using a strategy similar to that developed for vaccinia virus vectors $(22$, 27), AcMNPV-mediated expression of HSV1-tk resulted in conferred sensitivity of viral replication to nucleoside analogues, including Ganciclovir (28). Virus replication was restored when drug sensitivity was lost by replacement of $t k$ with a foreign gene, thereby allowing selective amplification of $t k^{-}$recombinants. In contrast to $t k$ that requires prior insertion of the marker into the virus genome, neo provides selectivity upon insertion that is not limited at a single locus in the AcMNPV genome.

\section{p35 as a dominant selectable marker for AcMNPV}

The high levels of BV production in SF21 cells by $p 35$-containing viruses compared to $p 35$-deletion mutants suggested that this virus-encoded gene would provide a powerful selection for recombinant viruses. This was first demonstrated by serial passage of virus mixtures in which $p 35$-containing viruses were selectivity amplified. In the most dramatic case, virus $\mathrm{v} \Delta 35 \mathrm{~K} / \mathrm{lacZ} / 35 \mathrm{~K}^{+}$was amplified more than two million-fold relative to a deletion mutant in only four passages (Fig. 5). These results also demonstrated that $p 35$-containing viruses could be amplified and subsequently identified within mixtures that contained more than a million-fold excess of virus lacking the gene. Thus, $p 35$ should be an effective marker for amplification and isolation of rare virus mutants. For purposes of generating virus mutants, $p 35$ has a distinct advantage because of its positionindependence that permits marker insertion at different sites 
within the AcMNPV genome. By using this approach, we have generated several mutants within the AcMNPV HindIII-K/Q region with allelic transfer plasmids that contain $p 35$ linked to altered virus sequences for replacement within the virus genome (29). The position-independent expression of $p 35$ may also be useful for studies of lepidopteran transposons that are capable of integrating within the AcMNPV genome $(1,24,30)$. By engineering such mobile elements to include $p 35$, it is expected that rare transposon insertions into $p 35$-deletion mutants can be detected and subsequently characterized. Studies are underway to test this possibility.

\section{p35 as a dominant selectable marker for the generation of AcMNPV expression vectors}

Compared to neo, $p 35$ provided dramatically higher levels of selective amplification. We have exploited this selectivity as a means to improve the recovery of recombinant virus vectors for foreign protein expression. When $p 35$ was incorporated into transfer plasmids containing a foreign gene (lacZ) under control of the polyhedrin promoter, approximately $15-30 \%$ of progeny obtained after transfection with deletion virus $v \Delta 35 \mathrm{~K}$ consisted of desired recombinant viruses in which the polyhedrin gene was replaced by the foreign gene (Table 2 ). This represented a 15to 300 -fold increase in the frequency $(0.1$ to $1 \%)$ with which recombinants are recovered using transfected (circular) viral DNA (4) and approximately the same as that obtained when viral DNA is linearized prior to transfection (20). By linearizing the DNA from $p 35$-deletion viruses, the proportion of desired recombinants increased even further, ranging from 82 to $96 \%$ (Table 3). This represents the highest fractional yield for desired recombinant plaques yet reported in the literature. By using liposome-mediated transfection methods, only small quantities of $p 35$-mutant DNA (100-200 ng) are required to obtain yields of $10^{3}$ to $10^{5}$ recombinant viruses per $\mathrm{mL}$ of growth medium when harvested 2 to 4 days after transfection. This conserves parental virus DNA which is produced at levels 10-fold lower than wild-type virus in $S$. frugiperda cells.

Several new methods for baculovirus expression vector construction are available. These include the use of endonucleaselinearized viral DNA $(20,31)$, the Cre-loxP recombination system (32), genome selection in Saccharomyces cerevisiae (33), and HSV1-tk-mediated drug selection (26). Use of allelic transfer plasmids that contain $p 35$ as a selectable marker provides an alternative and simple approach. The same cell line ( $S$. frugiperda SF21) can be used for transfection, purification of recombinant viruses, and production of foreign protein. Moreover, $p 35$-mediated selection does not involve potentially mutagenic drugs. Using $\beta$-galactosidase as a reporter, we have found that the presence of $p 35$ immediately adjacent to the polyhedrin promoter has little, if any, effect on the high level of foreign protein expression by AcMNPV vectors (8). In addition, the recovery rate of recombinant viruses is sufficiently high that a pure virus stock can be produced after a single round of plaque isolation (our unpublished results). This has reduced the time required to ascertain the level of foreign protein production to about 2-3 weeks as demonstrated by the construction of $p 35$-containing virus vectors by our own and other laboratories. A potentially useful feature of $p 35$ as a selectable marker is the relatively high rate $(\sim 30-50 \%)$ with which desired recombinant viruses are recovered when intact $\mathrm{BV}$ of the $p 35$-deletion mutant $\mathrm{v} \Delta 35 \mathrm{~K}$ is used in transfections rather than virus DNA. With appropriate modifications, it should be possible to eliminate the need for purified virus DNA, thereby simplifying transfections and reducing the expense of expression vector construction.

\section{ACKNOWLEDGMENTS}

We thank Julie A.Dickson and Pamela A.Hershberger for the construction of several viruses and plasmids used in this study. This work was supported in part by Public Health Service grant AI25557 from the National Institute of Allergy and Infectious Diseases. R.A.L. was supported by Viral Oncology Training Grant CA09075 from the National Institutes of Health.

\section{REFERENCES}

1. Blissard, G. W., and G. F. Rohrmann. (1990) Annu. Rev. Entomol. 35, 127-155.

2. Hawtin, R. E., and R. D. Possee. (1992) In N. Beckage, S. Thompson, and B. Federici (ed.), Parasites and Pathogens of Insects. Academic Press, Inc., N.Y. (in press).

3. Luckow, V. A., and M. D. Summers. (1988) Bio/Tech. 6:47-55.

4. O'Reilly, D. R., L. K. Miller, and V. A. Luckow. (1992) Baculovirus expression vectors: a laboratory manual. W. H. Freeman \& Co., Salt Lake City, Utah.

5. Wood, H. A., and R. R. Granados. (1991) Annu. Rev. Entomol. 45, 69-87.

6. Clem, R. J., M. Fechheimer, and L. K. Miller. (1991) Science 254, $1388-1390$

7. Friesen, P. D., and L. K. Miller. (1987) J. Virol. 61, 2265-2272.

8. Hershberger, P. A., J. A. Dickson, and P. D. Friesen. (1992) J. Virol. 66, $5525-5533$.

9. Davies, J., and A. Jiminez. (1980) Am. J. Trop. Med. Hyg. 29, 1089-1092.

10. Southern, P. J., and P. Berg. (1982) J. Mol. Appl. Genet. 1, 327-341.

11. Franke, C. A., C. M. Rice, J. H. Strauss, and D. E. Hruby. (1985) Mol. Cell. Biol. 5, 1918-1924.

12. Lee, M-A., and J. L. Yates. (1992) J. Virol. 66, 1899-1906.

13. Wang, F., A. Marchini, and E. Kieff. (1991) J. Virol. 65, 1701-1709.

14. Jarvis, D. L., J. G. W. Fleming, G. R. Kovacs, M. D. Summers, and L. A. Guarino. (1990) Bio/Tech. 8, 950-955.

15. Rio, D. C., and G. M. Rubin. (1985) Mol. Cell. Biol. 5, 1833-1838.

16. Vaughn, J. L., R. H. Goodwin, G. L. Thompkins, and P. McCawley. (1977) In Vitro (Rockville) 13, 213-217

17. Hink, W. F. (1970) Nature 225, 466-467.

18. Lee, H. H., and L. K. Miller. (1978) J. Virol. 27, 754-767.

19. Dickson, J. A., and P. D. Friesen. (1991) J. Virol. 65, 4006-4016.

20. Kitts, P. A., M. D. Ayres, and R. D. Possee. (1990) Nucl. Acids Res. 18, 5667-5672.

21. Nissen, M. S. and P. D. Friesen. (1989) J. Virol. 63, 493-503.

22. Ausubel, F. M., R. Brent, E. Kingston, D. D. Moore, J. G. Seidmen, J. A. Smith, and K. Struhl. (1989) Current Protocols in molecular biology. John Wiley \& Sons, Inc., N.Y.

23. Laemmli, U. K. (1970) Nature 227, 680-685.

24. Lerch, R. A., and P. D. Friesen. (1992) J. Virol. 66, 1590-1601.

25. Gonnet, P., and G. Devauchelle. (1987) C. R. Acad. Sci. Paris 305, $111-114$.

26. Godeau, F., C. Saucier, and P. Kourilsky. (1992) Nucl. Acids Res. 20, $6239-6246$.

27. Moss, B. (1991) Science 252, 1662-1667.

28. Syntex Laboratories, Inc. product monograph for Cytovene (Ganciclovir sodium), Palo Alto, CA, USA.

29. Rodems, S. M., J. A. Dickson, and P. D. Friesen (unpublished results).

30. Friesen, P. D. (1992) In N. Beckage, S. Thompson, and B. Federici (ed.), Parasites and Pathogens of Insects. Academic Press, Inc., N.Y. (in press).

31. Kitts, P. A. (1992) CLONTECHniques, Vol. VII, No. 2. Clonetech Laboratories, Inc.

32. Peakman, T. C., R. A. Harris, and D. R. Gewert. (1992) Nucl. Acid Res. 20, 495-500.

33. Patel, G., K. Nasmyth, and N. Jones. (1992) Nucl. Acid Res. 20, 97-104. 